\title{
Numerical Evaluation of 2-EGAOR Iterative Method on Image Blurring using Non-Linear Diffusion Equation
}

\author{
${ }^{1}$ Nurul Afiqah Basran, ${ }^{1}$ Jeng Hong Eng, ${ }^{2}$ Azali Saudi and ${ }^{1}$ Jumat Sulaiman \\ ${ }^{1}$ Faculty of Science and Natural Resources, \\ ${ }^{2}$ A Knowledge Technology Research Unit, Faculty of Computing and Informatics, \\ University of Malaysia Sabah (UMS), Jalan, 88400 Kota Kinabalu, Malaysia \\ nurulafiqahbasran@yahoo.com, +60148556550
}

\begin{abstract}
The application of non-linear diffusion equation for image blurring has become a significant study in image processing field. The process is carried out by smoothing the image while preserving the crucial part of the image, i.e., edge, shape and important features using non-linear diffusion equation. However, the classical iterative method to solve the equation requires high number of computations and make the filtering process slower. This study examined 2-EGAOR iterative method as an efficient solver to the image blurring as this method involves two point in one group to solve the linear system with two weighted parameter. For the performance comparison, the results of Successive Over-Relaxation (SOR), Accelerated Over-Relaxation (AOR) and 2-EGAOR iterative methods to develop the equivalent image producing by classical Jacobi method is recorded in this study. The number of iterations and computational time in solving the linear system are used as the evaluation criteria of these iterative methods. Based on the numerical experiment, the findings has shown the 2-EGAOR method able to blurred the image slightly faster as it gives the fewer number of iterations and computational time compared to the other mention methods.
\end{abstract}

Key words: Non-linear diffusion equation, image blurring, two point Explicit Group Accelerated Over-Relaxation (2-EGAOR), iteration, evaluation criteria, filtering process

\section{INTRODUCTION}

The wide application of Partial Difference Equations (PDEs) have become a crucial study to generate better algorithms and tools for image processing applications. The PDEs based image processing methods have been applied in various problems by past researchers, i.e., image editing ( $\mathrm{Yu}$ and Deng, 2016; Hong et al., 2017), edge detection (Dollar and Zitnick, 2015), image segmentation (Dhanachandra et al., 2015), image enhancement (Hama and Al-Ani, 2013), image smoothing (Li et al., 2018) and image denoising (Sharma et al., 2017). Apparently, the heat diffusion PDE is used in image blurring. However, the rate of diffusion in linear heat equation is constant across the whole image domain which causes the smoothing of the entire image. A solution to this problem is by utilizing the diffusion coefficient based on image gradients that can preserve edges information that are the solutions of nonlinear diffusion equations (Weickert et al., 1998; Dollar and Zitnick, 2015).
The idea of utilizing nonlinear diffusion equation in image processing was first initiated by Perona and Malik (1990) with controlling the rate of diffusion using local gradient magnitude function. The successful of the algorithm to solve many image processing problems has motivated Catte et al. (1992) on enhancing the edge preserving behaviour of Perona Malik model. An improved nonlinear diffusion algorithm was proposed by Wu and Zhong (2010) applied on image denoising problem. Noise is refers to the random signal that appears as random speckles which significantly corrupting the image quality. Therefore, this new method has been verified as an efficient method to properly denoise the images compared to the other existing methods as it able reduce image noise while maintain important details better by using wavelet coefficient.

Recently, Ma and Nie (2016) have proposed more advanced anisotropic diffusion filtering model for image denoising. The model applied characteristics and gradient variance parameter to classify the important image information such as edges, corners, smooth regions and

Corresponding Author: Nurul Afiqah Basran, Faculty of Science and Natural Resources, University of Malaysia Sabah (UMS), Jalan, 88400 Kota Kinabalu, Malaysia, nurulafiqahbasran@yahoo.com, +60148556550 
isolated noises. The differ between nonlinear diffusion and anisotropic diffusion are the diffusivity possess in anisotropic diffusion model is a tensor instead of scalar (Weickert, 1998). Then, the eigenvalues of diffusion tensor used by Ma and Nie (2016) are designed for adaptive diffusion from the different image information. Finally, an edge combination scheme is presented to maintain the edges after denoising by fusing the distinctive denoising and edge detection techniques.

Several other tools are also been proposed to solve image processing problems by using diffusion equation such discussed earlier in this study. However, the primary motivation of this study to evaluate the performance of iterative methods in solving nonlinear diffusion equation numerically. The focus of research is same as conducted by recent researcher on Poisson image blending problems (Hong et al., 2017; Eng et al., 2017a, b, 2018). Hence, this study utilized the potential of 2-EGAOR iterative method to solve the nonlinear diffusion equation for image blurring. Then, the findings of SOR and AOR iterative methods are determined to conduct comparison performances with 2-EGAOR iterative method. The performance of those iterative methods are examined by the number of iterations and the computational time taken.

The remains of the study is sorted out as follows. Section 2 introduces the nonlinear diffusion equation based on model developed by Perona and Malik (1990) and provide the formulation of iterative method using two-point Explicit Group Accelerated Over-Relaxation by Yousif and Evans (1986) and Martins et al. (2002) to solve the linear system.

Non-linear diffusion equation: The process of blurring an image using non-linear diffusion equation is one of the Partial Differential Equation (PDEs) based image filtering technique. This equation also can has widely been used in denoising (Chen et al., 2015), smoothing (Liu et al., 2017) and edge detection (Nomura et al., 2016) an image. According to Kamalaveni et al. (2015), this method improve the problem of linear diffusion filtering by avoiding the blurring the important features and localization. Equation 1 can be represent as follows (Perona and Malik, 1990):

$$
I_{t}=\operatorname{div}(g(x, y, t) \nabla I(x, y))
$$

Equation 1 apply the diffusion coefficient $\mathrm{g}(\mathrm{x}, \mathrm{y}, \mathrm{t})=\mathrm{c}(\|\mathrm{VI}(\mathrm{x}, \mathrm{y}, \mathrm{t})\|)$ that reduces the diffusivity at any point probable to be the edges. Based on the above equation, the symbol of div is known as the divergence operator. Meanwhile, VI represent the gradient magnitude operator respect to the spatial of $\mathrm{x}$ and $\mathrm{y}$. The diffusivities used from Eq. 1 is based on the diffusion coefficient c(.) given as Eq. 1:

$$
c(\nabla \mathrm{I})=\frac{1}{1+\left(\frac{\|\nabla \mathrm{I}\|}{\mathrm{K}}\right)^{2}}
$$

Equation 2 is known as edge stopping function that control the diffusivities suggested by Perona and Malik (1990). The diffusivity is enhancing in the interior of homogenous region while zero at the boundaries. Its means the blurring impact will be lessen at any location with high potential be the boundaries monitored by the local gradient magnitude function, $|\nabla| \mid$ where, $g(x, y, t)=$ $\mathrm{c}(|\mathrm{VI}|)$. Therefore, implicit finite difference discretization approach is applied in this study to form the approximate equation followed by linear systems and then solved by using 2-EGAOR.

\section{MATERIALS AND METHODS}

\section{Implementation of proposed iterative methods}

Point iterative methods: The discretization of Eq. 1 through finite difference method by using implicit scheme has been developed to form the approximation equation of standard five-point as in Eq. 3:

$$
\begin{aligned}
& \left(1+\lambda c_{N}+\lambda c_{S}+\lambda c_{E}+\lambda c_{W W}\right) U_{i, j, k+1}-\lambda c_{N} U_{i, j+1, k+1}- \\
& \lambda c_{S} U_{i, j-1, k+1}-\lambda c_{E} U_{i+1, j, k+1}-\lambda c_{W} U_{i-1, j, k+1} \cong U_{i, j, k}
\end{aligned}
$$

where, $\lambda=\Delta \mathrm{t} / \mathrm{h}^{2}$ as $\mathrm{h}=\Delta \mathrm{x}=\Delta \mathrm{y}$. To simplify Eq. 3, let $\beta=$ $1+\lambda \mathrm{c}_{\mathrm{N}}+\lambda \mathrm{c}_{\mathrm{S}}+\lambda \mathrm{c}_{\mathrm{E}}+\lambda \mathrm{c}_{\mathrm{W}}, \delta_{\mathrm{N}}=\lambda \mathrm{c}_{\mathrm{N}}, \delta_{\mathrm{S}}=\lambda \mathrm{c}_{\mathrm{S}}, \delta_{\mathrm{E}}=\lambda \mathrm{c}_{\mathrm{E}}$ and $\boldsymbol{\delta}_{\mathrm{W}}=$ $\lambda c_{W}$ then, developed Eq. 4 :

$$
\begin{aligned}
& \beta U_{i, j, k+1}-\delta_{N} U_{i, j+1, k+1}-\delta_{S} U_{i, j-1, k+1}{ }^{-} \\
& \delta_{E} U_{i+1, j, k+1}-\delta_{W W} U_{i-1, j, k+1} \cong U_{i, j, k} .
\end{aligned}
$$

The developed approximation (Eq. 4) consists of the sparse of linear system can be reconstruct in the matrix form composed as Eq. 5:

$$
\mathrm{A} \underline{\mathrm{U}}=\underline{\mathrm{b}}
$$

where, $\mathrm{A}$ and $\mathrm{b}$ are known and $\mathrm{U}$ is unknown. Since, the coefficient of the main diagonal is strictly dominant, the linear system (Eq. 5) comprises a unique solution (Young, 1954). Matrix A then be decomposed into three different matrices shown as Eq. 6: 


$$
\mathrm{A}=\mathrm{S}-\mathrm{T}-\mathrm{V}
$$

which the matrix of S refers to the diagonal part of matrix A while matrices of $-\mathrm{T}$ and $-\mathrm{V}$ are the strict lower triangular and strict upper triangular parts, respectively. Thus, the corresponding point SOR (Young, 1954) and AOR (Hadjidimos, 1978) iterative schemes in the matrix form as in Eq. 7:

$$
\begin{aligned}
& \mathrm{U}^{(\mathrm{n})}=(\mathrm{S}-\omega \mathrm{T})^{-1}[\omega \mathrm{V}+(1-\omega) \mathrm{S}] \mathrm{U}^{(\mathrm{n}-1)}+\omega(\mathrm{S}-\omega \mathrm{T})^{-1} \mathrm{~b} \\
& \mathrm{U}^{(\mathrm{n})}=(\mathrm{S}-\mathrm{rT})^{-1}[(1-\omega) \mathrm{S}+(\omega-\mathrm{r}) \mathrm{T}+\omega \mathrm{V}] \mathrm{U}^{(\mathrm{n}-1)}+\omega(\mathrm{S}-\mathrm{rT})^{-1} \mathrm{~b}
\end{aligned}
$$

for $\mathrm{n}=1,2,3$. The approximate (Eq. 4) also brings about the large linear system with sparse coefficient matrix which can be stated in algebraic Eq. 8 as:

$$
\sum_{j=1}^{m} a_{i, j} U_{j}=b_{i} ; \quad i=1,2,3, \cdots, m
$$

By applying the iterative methods of SOR and AOR (Basran et al., 2018) for solving Eq. 4 in the form of algebraic Eq. 8, the corresponding iteration scheme can be shown in Eq. 9:

$$
\begin{aligned}
& \mathrm{U}_{\mathrm{i}, \mathrm{j}}^{\mathrm{k}+1} \cong \frac{\omega}{\beta}\left(\mathrm{U}_{\mathrm{i}, \mathrm{j}}^{\mathrm{k}}+\delta_{\mathrm{N}} \mathrm{U}_{\mathrm{i}, \mathrm{j}+1}^{\mathrm{k}}+\delta_{\mathrm{S}} \mathrm{U}_{\mathrm{i}, \mathrm{j}-1}^{\mathrm{k}+1}+\delta_{\mathrm{E}} \mathrm{U}_{\mathrm{i}+1, \mathrm{j}}^{\mathrm{k}}+\delta_{\mathrm{W}} \mathrm{U}_{\mathrm{i}-1, \mathrm{j}}^{\mathrm{k}+1}\right)+ \\
& (1-\omega) \mathrm{U}_{\mathrm{i}, \mathrm{j}}^{\mathrm{k}} \\
& \mathrm{U}_{i, j}^{\mathrm{k}+1} \cong \frac{\mathrm{r}}{\beta}\left(\delta_{\mathrm{S}} \mathrm{U}_{i, j-1}^{\mathrm{k}+1}-\delta_{\mathrm{S}} \mathrm{U}_{i, j-1}^{\mathrm{k}}+\delta_{\mathrm{W}} \mathrm{U}_{\mathrm{i}-1, \mathrm{j}}^{\mathrm{k}+1}-\delta_{\mathrm{W}} \mathrm{U}_{\mathrm{i}-1, \mathrm{j}}^{\mathrm{k}}\right)+ \\
& \frac{\omega}{\beta}\left(U_{i, j}^{k}+\delta_{N} U_{i, j+1}^{k}+\delta_{S} U_{i, j-1}^{k}+\delta_{E} U_{i+1, j}^{k}+\delta_{W} U_{i-1, j}^{k}\right)+(1-\omega) U_{i, j}^{k}
\end{aligned}
$$

for $\mathrm{k}=1,2,3, \ldots, \mathrm{n}$. The SOR iterative method can be reconstruct from $\mathrm{AOR}$ method by placing the value of equivalent to $\omega$. The iteration process of Eq. 9 continues until the convergence criterion is satisfied which in this study we imply the convergence error tolerance when the overall pixel difference between the images produced by using Jacobi iterative methods which is $<5.0 \%$. The weighted parameter, $\omega$ is set from the range of $1<\omega<2$. The optimal value of the weighted parameter, $\omega$ can be obtained through several test that gives the least number of iterations, $k$. Then, choosing the consecutive values of $\mathrm{r}$ with precision 0.01 within the interval 0.1 for AOR method.

Study conducted by Dhanachandra et al. (2015) has used the Gauss-Seidel iterative method as the faster solver than the Jacobi iterative method. Besides, recent studied in image processing also has employed block iterative method for solving Poisson image blending (Eng et al., 2017a, b, 2018). Other than that block iterative methods also had widely been studied in robot path planning problems (Saudi et al., 2017; Dahalan et al., 2017) and other numerical problems (Akhir and Sulaiman, 2015; Chew and Sulaiman, 2017).

Two point explicit group accelerated over-relaxation iterative method: Two point explicit group iterative has been constructed to solve the linear system of Eq. 4 where two node points as shown in Eq. 10 are applied form two-point block such:

$$
\begin{aligned}
& \mathrm{U}_{i, j}^{\mathrm{k}+1} \cong \frac{1}{\beta}\left(\mathrm{U}_{\mathrm{i}, \mathrm{j}}^{\mathrm{k}}+\delta_{\mathrm{N}} \mathrm{U}_{\mathrm{i}, \mathrm{j}+1}^{\mathrm{k}}+\delta_{\mathrm{S}} \mathrm{U}_{\mathrm{i}, \mathrm{j}-1}^{\mathrm{k}+1}+\delta_{\mathrm{E}} \mathrm{U}_{\mathrm{i}+1, j}^{\mathrm{k}}+\delta_{\mathrm{W}} \mathrm{U}_{\mathrm{i}-1, \mathrm{j}}^{\mathrm{k}+1}\right) \\
& \mathrm{U}_{\mathrm{i}+1, \mathrm{j}}^{\mathrm{k}+1} \cong \frac{1}{\beta_{1}}\left(\mathrm{U}_{\mathrm{i}+1, j}^{\mathrm{k}}+\delta_{\mathrm{N} 1} \mathrm{U}_{\mathrm{i}+1, \mathrm{j}+1}^{\mathrm{k}}+\delta_{\mathrm{S} 1} \mathrm{U}_{\mathrm{i}+1, j-1}^{\mathrm{k}+1}+\delta_{\mathrm{E} 1} \mathrm{U}_{\mathrm{i}+2, j}^{\mathrm{k}}+\delta_{\mathrm{W} 1} \mathrm{U}_{\mathrm{i}, \mathrm{j}}^{\mathrm{k}+1}\right)
\end{aligned}
$$

Equation 10 above can be formulated into matrix form of Eq. 5 which constructed as:

$$
\left[\begin{array}{cc}
\beta & -\delta_{\mathrm{E}} \\
-\delta_{W 1} & \beta_{1}
\end{array}\right]\left[\begin{array}{c}
\mathrm{U}_{\mathrm{i}, j}^{\mathrm{k}+1} \\
\mathrm{U}_{\mathrm{i}+1, j}^{\mathrm{k}+1}
\end{array}\right]=\left[\begin{array}{c}
\mathrm{U}_{\mathrm{i}, \mathrm{j}}^{\mathrm{k}}+\delta_{\mathrm{N}} \mathrm{U}_{\mathrm{i}, \mathrm{j}+1}^{\mathrm{k}}+\delta_{\mathrm{S}} \mathrm{U}_{\mathrm{i}, \mathrm{j}-1}^{\mathrm{k}+1}+\delta_{\mathrm{W}} \mathrm{U}_{\mathrm{i}-1, \mathrm{j}}^{\mathrm{k}+1} \\
\mathrm{U}_{\mathrm{i}+1, j}^{\mathrm{k}}+\delta_{\mathrm{N} 1} \mathrm{U}_{\mathrm{i}+1, \mathrm{j}+1}^{\mathrm{k}}+\delta_{\mathrm{S} 1} \mathrm{U}_{\mathrm{i}+1, j-1}^{\mathrm{k}+1}+\delta_{\mathrm{E} 1} \mathrm{U}_{\mathrm{i}+2, \mathrm{j}}^{\mathrm{k}}
\end{array}\right]
$$

Then, the inverse matrix of the coefficient lattice of Eq. 11 need to be identified in order to form a general scheme of two point Explicit Group (2-EG) iterative method. Hence, the general scheme of 2-EG can be shown as below (Yousif and Evans, 1986):

$$
\begin{aligned}
& {\left[\begin{array}{c}
\mathrm{U}_{i, j}^{\mathrm{k}+1} \\
\mathrm{U}_{\mathrm{i}+1, j}^{\mathrm{k}+1}
\end{array}\right]=\frac{1}{\beta \beta_{1}-\delta_{\mathrm{E}} \delta_{\mathrm{W} 1}}\left[\begin{array}{cc}
\beta_{1} & \delta_{\mathrm{E}} \\
\delta_{W 1} & \beta
\end{array}\right]} \\
& {\left[\begin{array}{c}
\mathrm{U}_{i, j}^{\mathrm{k}}+\delta_{\mathrm{N}} \mathrm{U}_{i, j+1}^{\mathrm{k}}+\delta_{\mathrm{S}} \mathrm{U}_{i, j-1}^{\mathrm{k}+1}+\delta_{W} \mathrm{U}_{\mathrm{i}-1, j}^{\mathrm{k}+1} \\
\mathrm{U}_{\mathrm{i}+1, j}^{\mathrm{k}}+\delta_{\mathrm{N} 1} \mathrm{U}_{\mathrm{i}+1, \mathrm{j}+1}^{\mathrm{k}}+\delta_{\mathrm{S} 1} \mathrm{U}_{\mathrm{i}+1, j-1}^{\mathrm{k}+1}+\delta_{\mathrm{E} 1} \mathrm{U}_{\mathrm{i}+2, j}^{\mathrm{k}}
\end{array}\right]}
\end{aligned}
$$

We can simplify Eq. 13 as following:

$$
\begin{aligned}
& \mathrm{U}_{\mathrm{i}, \mathrm{j}}^{\mathrm{k}+1}=\frac{1}{\beta \beta_{1}-\delta_{\mathrm{E}} \delta_{W 1}}\left(\beta_{1} \mathrm{~S}_{1}+\delta_{\mathrm{E}} \mathrm{S}_{2}\right) \\
& \mathrm{U}_{\mathrm{i}+1, \mathrm{j}}^{\mathrm{k}+1}=\frac{1}{\beta \beta_{1}-\delta_{\mathrm{E}} \delta_{W}}\left(\delta_{W 1} \mathrm{~S}_{1}+\beta \mathrm{S}_{2}\right)
\end{aligned}
$$

where, $\mathrm{S}_{1}$ and $\mathrm{S}_{1}$ denoted as Eq. 14:

$$
\begin{aligned}
& \mathrm{S}_{1}=\mathrm{U}_{\mathrm{i}, \mathrm{j}}^{\mathrm{k}}+\delta_{\mathrm{N}} \mathrm{U}_{\mathrm{i}, \mathrm{j}+1}^{\mathrm{k}}+\delta_{\mathrm{S}} \mathrm{U}_{\mathrm{i}, \mathrm{j}-1}^{\mathrm{k}+1}+\delta_{\mathrm{W}} \mathrm{U}_{\mathrm{i}-1, \mathrm{j}}^{\mathrm{k}+1} \\
& \mathrm{~S}_{2}=\mathrm{U}_{\mathrm{i}+1, j}^{\mathrm{k}}+\delta_{\mathrm{N} 1} \mathrm{U}_{\mathrm{i}+1, \mathrm{j}+1}^{\mathrm{k}}+\delta_{\mathrm{S} 1} \mathrm{U}_{\mathrm{i}+1, \mathrm{k}-1}^{\mathrm{k}+1}+\delta_{\mathrm{E} 1} \mathrm{U}_{\mathrm{i}+2, \mathrm{j}}^{\mathrm{k}}
\end{aligned}
$$




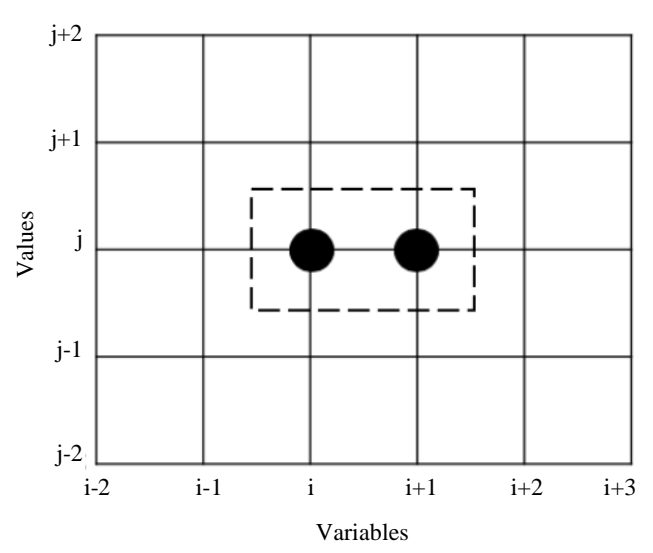

Fig. 1: The 2-EGAOR iterative method computational network in finite grid

It can be seen that, the calculations for both points $\mathrm{U}_{i, j}^{k+1}$ and $\mathrm{U}_{i+1, j}^{k+1}$ are totally independent. As shown in Fig. 1, the implementation of 2-EG iterative method occurs in each group of two node points meanwhile the ungroup nodes positioned next to the boundary are computed using direct method (Yousif and Evans, 1986). To form 2-EGAOR iterative method, the weighted parameter of $r$ and $\omega$ need to be added into the Eq. 14. Then, it can be rewritten as Martins et al. (2002) (Eq. 15):

$$
\begin{aligned}
\mathrm{U}_{i, j}^{\mathrm{k}+1}= & \frac{\mathrm{r}}{\beta \beta_{1}-\delta_{\mathrm{E}} \delta_{\mathrm{W} 1}}\left(\mathrm{~T}_{1}\right)+\frac{\omega}{\beta \beta_{1}-\delta_{\mathrm{E}} \delta_{\mathrm{W} 1}}\left(\beta_{1} \mathrm{~S}_{1 \mathrm{NEW}}+\delta_{\mathrm{E}} \mathrm{S}_{2 \mathrm{NEW}}\right)+ \\
\mathrm{U}_{\mathrm{i}+1, j}^{\mathrm{k}+1}= & \frac{\mathrm{r}}{\beta \beta_{1}-\delta_{\mathrm{E}} \delta_{\mathrm{W} 1}}\left(\mathrm{~T}_{2}\right)+\frac{\omega}{\beta \beta_{1}-\delta_{\mathrm{E}} \delta_{\mathrm{W} 1}}\left(\delta_{\mathrm{W} 1} \mathrm{~S}_{1 \mathrm{NEW}}+\beta \mathrm{S}_{2 \mathrm{NEW}}\right)+ \\
& (1-\omega) \mathrm{U}_{\mathrm{i}+1, j}^{\mathrm{k}}
\end{aligned}
$$

The symbols of $\mathrm{S}_{1 \mathrm{NEW}}, \mathrm{S}_{2 \mathrm{NEW}}, \mathrm{T}_{1}$ and $\mathrm{T}_{2}$ in Eq. 14 can be present as in Eq. 16 and 17:

$$
\begin{aligned}
& \mathrm{S}_{1 \mathrm{NEW}}=\mathrm{U}_{\mathrm{i}, \mathrm{j}}^{\mathrm{k}}+\delta_{\mathrm{N}} \mathrm{U}_{\mathrm{i}, \mathrm{j}+1}^{\mathrm{k}}+\delta_{\mathrm{S}} \mathrm{U}_{\mathrm{i}, \mathrm{j}-1}^{\mathrm{k}}+\delta_{\mathrm{WW}} \mathrm{U}_{\mathrm{i}-1, \mathrm{j}}^{\mathrm{k}} \\
& \mathrm{S}_{2 \mathrm{NEW}}=\mathrm{U}_{\mathrm{i}+1, j}^{\mathrm{k}}+\delta_{\mathrm{N} 1} \mathrm{U}_{\mathrm{i}+1, j+1}^{\mathrm{k}}+\delta_{\mathrm{S} 1} \mathrm{U}_{\mathrm{i}+1, j-1}^{\mathrm{k}}+\delta_{\mathrm{E} 1} \mathrm{U}_{\mathrm{i}+2, \mathrm{j}}^{\mathrm{k}} \\
& \mathrm{T}_{1}=\beta_{1}\left(\delta_{\mathrm{S}} \mathrm{U}_{\mathrm{i}, \mathrm{j}-1}^{\mathrm{k}+1}-\delta_{\mathrm{S}} \mathrm{U}_{\mathrm{i}, \mathrm{j}-1}^{\mathrm{k}}+\delta_{\mathrm{W}} \mathrm{U}_{\mathrm{i}-1, \mathrm{j}}^{\mathrm{k}+1}-\delta_{\mathrm{W}} \mathrm{U}_{\mathrm{i}-1, \mathrm{j}}^{\mathrm{k}}\right)+ \\
& \delta_{\mathrm{E}}\left(\delta_{\mathrm{S} 1} \mathrm{U}_{\mathrm{i}+1, j-1}^{\mathrm{k}+1}-\delta_{\mathrm{S} 1} \mathrm{U}_{\mathrm{i}+1, j-1}^{\mathrm{k}}\right) \\
& \mathrm{T}_{2}=\delta_{\mathrm{W} 1}\left(\delta_{\mathrm{S}} \mathrm{U}_{\mathrm{i}, \mathrm{j}-1}^{\mathrm{k}+1}-\delta_{\mathrm{S}} \mathrm{U}_{\mathrm{i}, \mathrm{j}-1}^{\mathrm{k}}+\delta_{\mathrm{W}} \mathrm{U}_{\mathrm{i}-1, \mathrm{j}}^{\mathrm{k}+1}-\delta_{\mathrm{W}} \mathrm{U}_{\mathrm{i}-1, \mathrm{j}}^{\mathrm{k}}\right)+ \\
& \beta\left(\delta_{\mathrm{S} 1} \mathrm{U}_{\mathrm{i}+1, j-1}^{\mathrm{k}+1}-\delta_{\mathrm{S} 1} \mathrm{U}_{\mathrm{i}+1, j-1}^{\mathrm{k}}\right)
\end{aligned}
$$

\section{RESULTS AND DISCUSSION}

The developed linear system from approximation non-linear diffusion equation is then solved by 2-EGAOR.
The performance of this iterative method is compared with SOR and AOR methods. The assessment is done by contrasting the quantity of iteration and computational time taken of these iterative techniques in delivering nearly a similar impact of blurred image. In order to produce almost the same blurred image for each methods, we set up the classical Jacobi iterative method as the control method. The iterations of SOR, AOR and 2-EGAOR are stopped when the overall pixels difference to the output image of Jacobi $<5 \%$. The blurring rate is controlled by diffusion coefficient function as mention in section 2. So, this experiment used the threshold value at $\mathrm{K}=5$ for Eq. 2 and time-step or value of $\lambda$ in Eq. 3 at 1.0. This study used three example pictures as shown in Fig. 2 for the blurring process as arranged accordingly, to the image sizes of $512 \times 512,1024 \times 1024$ and $2048 \times 2048$, respectively.

The number of iterations and blurring time are examined to compare the efficiency of 2-EGAOR iterative method against SOR and AOR methods. The iterations are running three times for colour (Red, Green and Blue) channels separately as the algorithm filtered the colour images. This causing each colour recorded different number of iterations $\mathrm{k}$ and computational time t. Thus, the average number of iterations $\mathrm{k}$ and computational time $\mathrm{t}$ of the three channels run for each example, images are taken and recorded in Fig. 1 and 2, respectively. For Jacobi method, the number of iterations used for the control parameter of the final image in this experiment are $\mathrm{k}=200$, $\mathrm{k}=400$ and $\mathrm{k}=800$, for example (a-c), respectively.

By referring to Table 1, 2-EGAOR iterative methods significantly reduce the number of iterations compared to $\mathrm{SOR}$ and $\mathrm{AOR}$ in producing the blurred images. The

\begin{tabular}{|c|c|c|c|c|c|c|c|}
\hline \multirow{3}{*}{$\begin{array}{l}\text { Examples/ } \\
\text { Methods }\end{array}$} & \multirow{3}{*}{$\begin{array}{c}\text { Jacobi } \\
-\mathrm{k} \\
\end{array}$} & \multicolumn{2}{|c|}{ SOR } & \multicolumn{2}{|c|}{ AOR } & \multicolumn{2}{|c|}{ 2-EGAOR } \\
\hline & & --- & ----- & & ------ & --- & ------ \\
\hline & & $\mathrm{k}$ & error & $\mathrm{k}$ & error & $\mathrm{k}$ & error \\
\hline $512 \times 512$ & 200 & 17 & 0.0477 & 14 & 0.0475 & 13 & 0.0468 \\
\hline $1024 \times 1024$ & 400 & 50 & 0.0483 & 39 & 0.0492 & 37 & 0.0481 \\
\hline $2048 \times 2048$ & 800 & 79 & 0.0495 & 61 & 0.0494 & 58 & 0.0494 \\
\hline
\end{tabular}
2-EGAOR iterative method requires the high percentage of iterations reduction by $90.75-93.5 \%$ against Jacobi method. Meanwhile, the method slightly better in reducing the number of iterations by $23.53-26.58 \%$ and 4.91-7.14\% against SOR and AOR iterative methods, respectively. Thereby, the result of the blurring time, $t$ in Table 2 also proved that the 2-EGAOR method able to

Table 1: The number of iteration, $\mathrm{k}$ for image blurring by SOR, AOR and 2-EGAOR iterative methods

Table 2: The computational time, $t$ (milliseconds) for image blurring by SOR, AOR and 2-EGAOR iterative methods

\begin{tabular}{lllll}
\hline Example/Method & Jacobi & SOR & AOR & 2-EGAOR \\
\hline $512 \times 512$ & 12746 & 1349 & 1223 & 1167 \\
$1024 \times 1024$ & 188082 & 19546 & 16289 & 16086 \\
$2048 \times 2048$ & 2220414 & 176664 & 166180 & 151825 \\
\hline
\end{tabular}


(a)

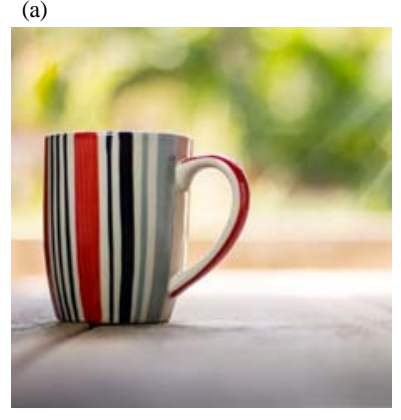

(b)

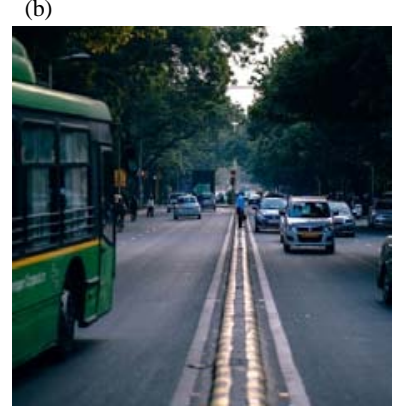

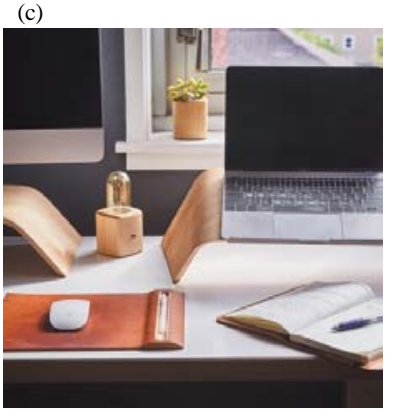

Fig. 2: a-c) The input images

(a)

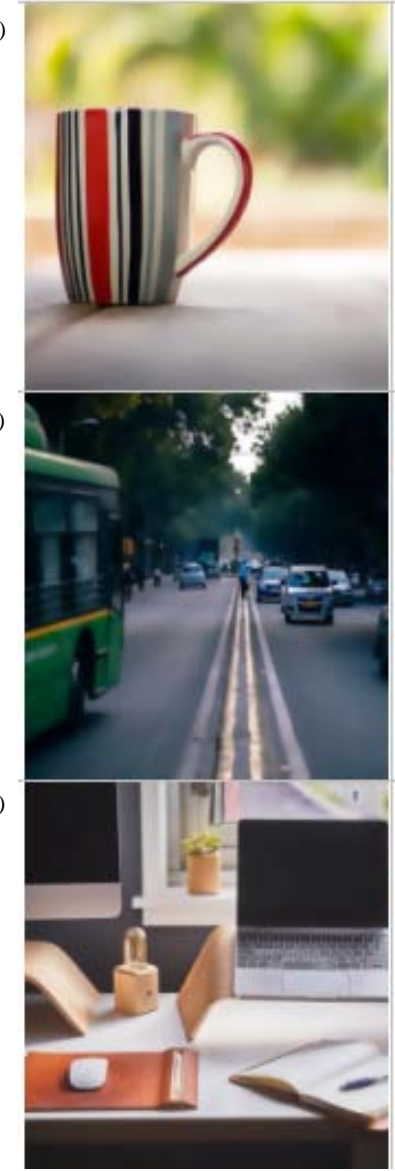

(i)

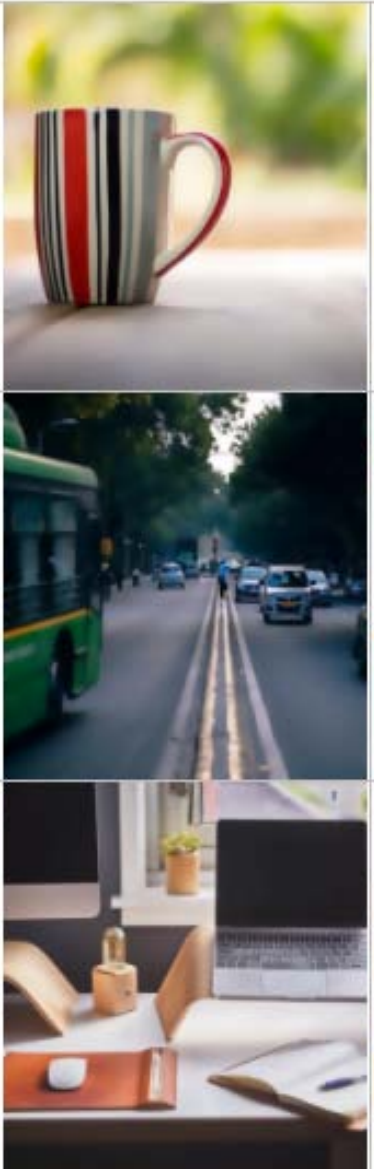

(ii)

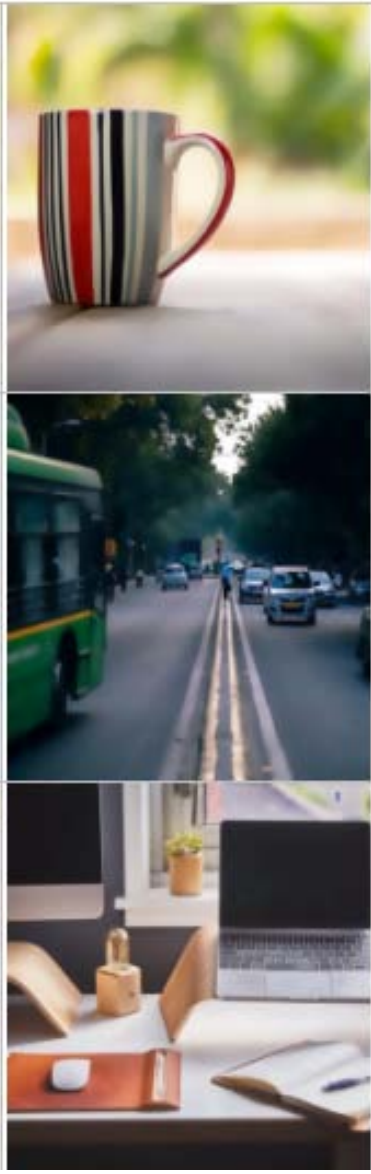

(iii)

Fig. 3: The output image produced by (i) SOR (ii) AOR and (iii) 2-EGAOR iterative methods using examples (a-c)

produce the faster blurred images as it requires the shortest time compared to SOR and AOR methods. By using 2-EGAOR iterative method, the blurring time taken improved by approximately $90.84-93.16 \%$ compared to Jacobi method. By comparing to the others iterative methods, the 2-EGAOR has reduced the blurring time by $13.49-17.70 \%$ and $4.58-8.64 \%$, respectively.
Based on the numerical result, the 2-EGAOR iterative method acquires minimum iterations and blurring time compared to other methods. This is because 2-EGAOR is block iterative method where a group of two points is calculated in one iteration. There is no obvious difference for the blurred images produced by all three methods as illustrated in Fig. 3 which the pixels difference (error) 
between image produced by Jacobi method and those three iterative methods are $<5 \%$ as shown in Table 1.

\section{CONCLUSION}

This study attempt on utilizing the 2-EGAOR technique in image blurring has been obtained and discussed. We have compared the number of iterations and computational time for the image to blur of 2-EGAOR iterative method in solving the proposed application with SOR and AOR iterative methods. The Jacobi has used as a control method with iterations, $\mathrm{k}=200, \mathrm{k}=400$ and $\mathrm{k}=800$, for example (a-c), respectively which based on the size of image stated earlier. The iterations have stopped if the pixels difference of the blurred images produce from each methods against output images of Jacobi method is $<5 \%$. As expected, the results have showed the 2-EGAOR iterative method is superior to SOR and $\mathrm{AOR}$ with the smallest iterations and computational time in producing the same quality of Jacobi image. The reduction percentage for both criteria has slightly reduced approximately by more than 13.00 and $4.00 \%$ for SOR and AOR methods, respectively. Besides, the performance of the 2-EGAOR iterative method which is categorized as a family of two point block and two parameter iterative method has been analyse in this study. Then, future research we will be continued to inquire the efficiency of the four point block such as 4-EGSOR (Eng et al. 2017a, b; Chew and Sulaiman, 2017), 4-EGAOR (Akhir and Sulaiman, 2015; Dahalan et al., 2017) and also half sweep method (Chew and Sulaiman, 2018) that involves half of the grid points in the iteration process.

\section{ACKNOWLEDGEMENT}

For this research, the authors would like to acknowledge Universiti Malaysia Sabah research grant (GUG0223-1/2018) upon support for this publication.

\section{REFERENCES}

Akhir, M.K.M. and J. Sulaiman, 2015. The 4-EGAOR method for solving triangle element approximation of 2D poisson equations. Appl. Math. Sci., 9: 5561-5571.

Basran, N.A., J.H. Eng, A. Saudi and J. Sulaiman, 2018. Numerical analysis of AOR iteration for anisotropic diffusion equation in image blurring. AIP. Conf. Proc., Vol. 2013, 10.1063/1.5054239

Catte, F., P.L. Lions, J.M. Morel and T. Coll, 1992. Image selective smoothing and edge detection by nonlinear diffusion. SIAM. J. Numer. Anal., 29: 182-193.
Chen, Y., W. Yu and T. Pock, 2015. On learning optimized reaction diffusion processes for effective image restoration. Proceedings of the IEEE International Conference on Computer Vision and Pattern Recognition, June 7-12, 2015, IEEE, Boston, Massachusetts, USA., ISBN:978-1-4673-6964-0, pp: 5261-5269.

Chew, J.V.L. and J. Sulaiman, 2017. Application of four-point newton-EGSOR iteration for the numerical solution of 2D Porous Medium Equations. J. Phys. Conf. Ser., 890: 1-7.

Chew, J.V.L. and J. Sulaiman, 2018. Implicit finite difference solution of one-dimensional porous medium equations using half-sweep newton-explicit group iterative method. J. Eng. Appl. Sci., 13: 1286-1290.

Dahalan, A.A., A. Saudi, J. Sulaiman and W.R.W. Din, 2017. Numerical evaluation of mobile robot navigation in static indoor environment via EGAOR Iteration. J. Phys. Conf. Ser., 890: 01 2064-012064.

Dhanachandra, N., K. Manglem and Y.J. Chanu, 2015. Image segmentation using k-means clustering algorithm and subtractive clustering algorithm. Procedia Comput. Sci., 54: 764-771.

Dollar, P. and C.L. Zitnick, 2015. Fast edge detection using structured forests. IEEE. Trans. Pattern Anal. Mach. Intell., 37: 1558-1570.

Eng, J.H., A. Saudi and J. Sulaiman, 2017a. Numerical analysis of the explicit group iterative method for solving poisson image blending problem. Intl. J. Imaging Rob., 17: 15-24.

Eng, J.H., A. Saudi and J. Sulaiman, 2017b. Numerical evaluation of solving poisson image blending problem by four-point EGSOR iterative method. Far East J. Math. Sci., 102: 3127-3139.

Eng, J.H., A. Saudi and J. Sulaiman, 2018. Performance analysis of the explicit decoupled group iteration via five-point rotated Laplacian operator in solving poisson image blending problem. Indian J. Sci. Technol., 11: 1-6.

Hadjidimos, A., 1978. Accelerated overrelaxation method. Math. Comput., 32: 149-157.

Hama, S.M. and M.S. Al-Ani, 2013. Medical image enhancement based on an efficient approach for adaptive anisotropic diffusion. Intl. J. Adv. Eng. Technol., 6: 1424-1430.

Hong, E.J., A. Saudi and J. Sulaiman, 2017. Application of SOR iteration for poisson image blending. Proceedings of the International Conference on High Performance Compilation, Computing and Communications (HP3C-2017), March 22-24, 2017, ACM,New York, USA., ISBN:978-1-4503-4868-3,pp: 60-64. 
Kamalaveni, V., R. Anitha Rajalakshmi and K.A. Narayanankutty, 2015. Image denoising using variations of perona-malik with different edge stopping functions. Procedia Comput. Sci., 58: 673-682.

Li, Y., F. Liu, I.W. Turner and T. Li, 2018. Time-fractional diffusion equation for signal smoothing. Appl. Math. Comput., 326: 108-116.

Liu, C., X. Yan and B. Yang, 2017. An adaptive anisotropic thermal diffusion filter for image smoothing. Proceedings of the 2017 10th International Congress on Image and Signal Processing, BioMedical Engineering and Informatics (CISP-BMEI), October 14-16, 2017, IEEE, Shanghai, China, ISBN:978-1-5386-1938-4, pp: 1-5.

$\mathrm{Ma}, \mathrm{H}$. and Y. Nie, 2016. An edge fusion scheme for image denoising based on anisotropic diffusion models. J. Visual Commun. Image Represent., 40: 406-417.

Martins, M.M., W.S. Yousif and D.J. Evans, 2002. Explicit group AOR method for solving elliptic partial differential equations. Neural Parallel Sci. Comput., 10: 411-422.

Nomura, A., K. Okada and Y. Mizukami, 2016. Preprocessing in a reaction-diffusion algorithm designed for image edge detection. Proceedings of the 2016 IEEE International Symposium on Signal Processing and Information Technology (ISSPIT), December 12-14, 2016, IEEE, Limassol, Cyprus, ISBN: 978-1-5090-5845-7, pp: 166-171.

Perona, P. and J. Malik, 1990. Scale-space and edge detection using anisotropic diffusion. IEEE Trans. Pattern Anal. Mach. Intell., 12: 629-639.
Saudi, A., J. Sulaiman and S.H. Tanalol, 2017. Path planning in structured environment using harmonic potential fields via block iterative method. Proceedings of the 5th International Conference on Future Computational Technologies (ICFCT'2017), April 18-19, 2017, Kyoto, Japan, pp: 99-106.

Sharma, P., K. Khan and K. Ahmad, 2017. Image denoising method based on local neighbourhood and modified mean in wavelet packet domain. Global Sci. Tech., 9: 129-137.

Weickert, J., 1998. Anisotropic Diffusion in Image Processing. Vol. 1, Teubner, Stuttgart, Germany, Pages: 184 .

Weickert, J., B.M. Romeny and M.A. Viergever, 1998. Efficient and reliable schemes for nonlinear diffusion filtering. IEEE Trans. Image Process., 7: 398-410.

$\mathrm{Wu}, \mathrm{W}$. and $\mathrm{C}$. Zhong, 2010. An improved nonlinear diffusion algorithm for image denoising. Proceedings of the 2010 International Conference on Computer, Mechatronics, Control and Electronic Engineering Vol. 5, August 24-26, 2010, IEEE, Changchun, China, ISBN:978-1-4244-7957-3, pp: 188-191.

Young, D., 1954. Iterative methods for solving partial difference equations of elliptic type. Trans. Am. Math. Soc., 76: 92-111.

Yousif, W.S. and D.J. Evans, 1986. Explicit group over-relaxation methods for solving elliptic partial differential equations. Math. Comput. Simulat., 28: 453-466.

$\mathrm{Yu}, \mathrm{H}$. and L.J. Deng, 2016. Image Editing Via Searching Source Image. In: Applied Mechanics, Mechatronics and Intelligent Systems, Qin, S. and X. Li (Eds.). World Scientific, Singapore, ISBN: 789814733878 , pp: 373-380. 